\title{
Rational design of Lepidoptera-specific insecticidal inhibitors targeting farnesyl diphosphate synthase, a key enzyme of the juvenile hormone biosynthetic pathway
}

\author{
Marie-Ève Picard, ${ }^{1, *}$ Michel Cusson, ${ }^{1,2}$ Stephanie E. Sen ${ }^{3}$ and Rong Shi ${ }^{1}$ \\ ${ }^{1}$ Département de biochimie, de microbiologie et de bio-informatique, Institut de Biologie Intégrative et des Systèmes, PROTEO, Université Laval, Quebec \\ City, QC, GIV 0A6, Canada \\ ${ }^{2}$ Natural Resources Canada, Canadian Forest Service, Laurentian Forestry Centre, 1055 du P.E.P.S., P.O. Box 10380, Station Ste. Foy, Quebec City, QC, G1V \\ 4C7, Canada \\ ${ }^{3}$ Department of Chemistry, The College of New Jersey, P.O. Box 7718, Ewing, NJ 08628, USA
}

(Received November 13, 2020; Accepted December 22, 2020)

\begin{abstract}
Reducing the use of broad-spectrum insecticides is one of the many challenges currently faced by insect pest management practitioners. For this reason, efforts are being made to develop environmentally benign pest-control products through bio-rational approaches that aim at disrupting physiological processes unique to specific groups of pests. Perturbation of hormonal regulation of insect development and reproduction is one such strategy. It has long been hypothesized that some enzymes in the juvenile hormone biosynthetic pathway of moths, butterflies and caterpillars (order Lepidoptera) display unique structural features that could be targeted for the development of Lepidoptera-specific insecticides, a promising avenue given the numerous agricultural and forest pests belonging to this order. Farnesyl diphosphate synthase, FPPS, is one such enzyme, with recent work suggesting that it has structural characteristics that may enable its selective inhibition. This review synthesizes current knowledge on FPPS and summarizes recent advances in its use as a target for insecticide development.
\end{abstract}

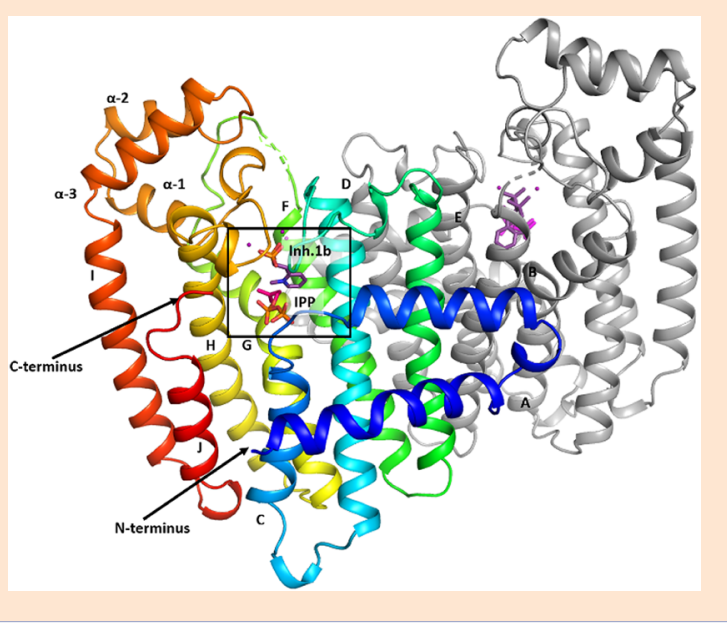

Keywords: farnesyl diphosphate synthase, short-chain isoprenyl diphosphate synthases, juvenile hormone, Lepidoptera, enzyme inhibitors, bisphosphonate.

\section{Introduction}

Farnesyl diphosphate synthase (FPPS) is a ubiquitous enzyme belonging to the mevalonate pathway (MVP). It catalyzes the sequential head-to-tail condensation of isoprene $\left(\mathrm{C}_{5}\right)$ units to form the sesquiterpene $\left(\mathrm{C}_{15}\right)$ farnesyl diphosphate (FPP), which is a precursor to several MVP-related end-products, including

\footnotetext{
* To whom correspondence should be addressed.

E-mail: marie-eve.picard.8@ulaval.ca

Published online January 28, 2021
}

(c) BY-NC-ND $\odot$ Pesticide Science Society of Japan 2021. This is an open access article distributed under the Creative Commons Attribution-NonCommercial-NoDerivatives 4.0 International (CC BY-NC-ND 4.0) License (https://creativecommons.org/licenses/by-nc-nd/4.0/) the "juvenile hormones" (JH) in insects. In a famous paper published in Scientific American, Williams ${ }^{1)}$ predicted the advent of a "third-generation" of insecticides whose modes of action would result in the disruption of hormonal regulation. In this context, JH seemed like an attractive target as it is found almost exclusively in insects and plays major roles in both development and reproduction. ${ }^{2,3)}$ Indeed, JH-related molecules (JH analogs) have since been developed and used as insecticides for the control of various insects, particularly those considered pests as adults. $^{4)}$

$\mathrm{JH}$ is produced and released into the hemolymph by paired endocrine glands known as the corpora allata (CA). ${ }^{5,6)}$ It is involved in various aspects of reproduction in adult insects, including ovarian maturation and pheromone production. ${ }^{7}$ During larval molts, high levels of JH inhibit the early onset of metamorphosis. Shortly after the final larval molt, JH titers drop significantly, 


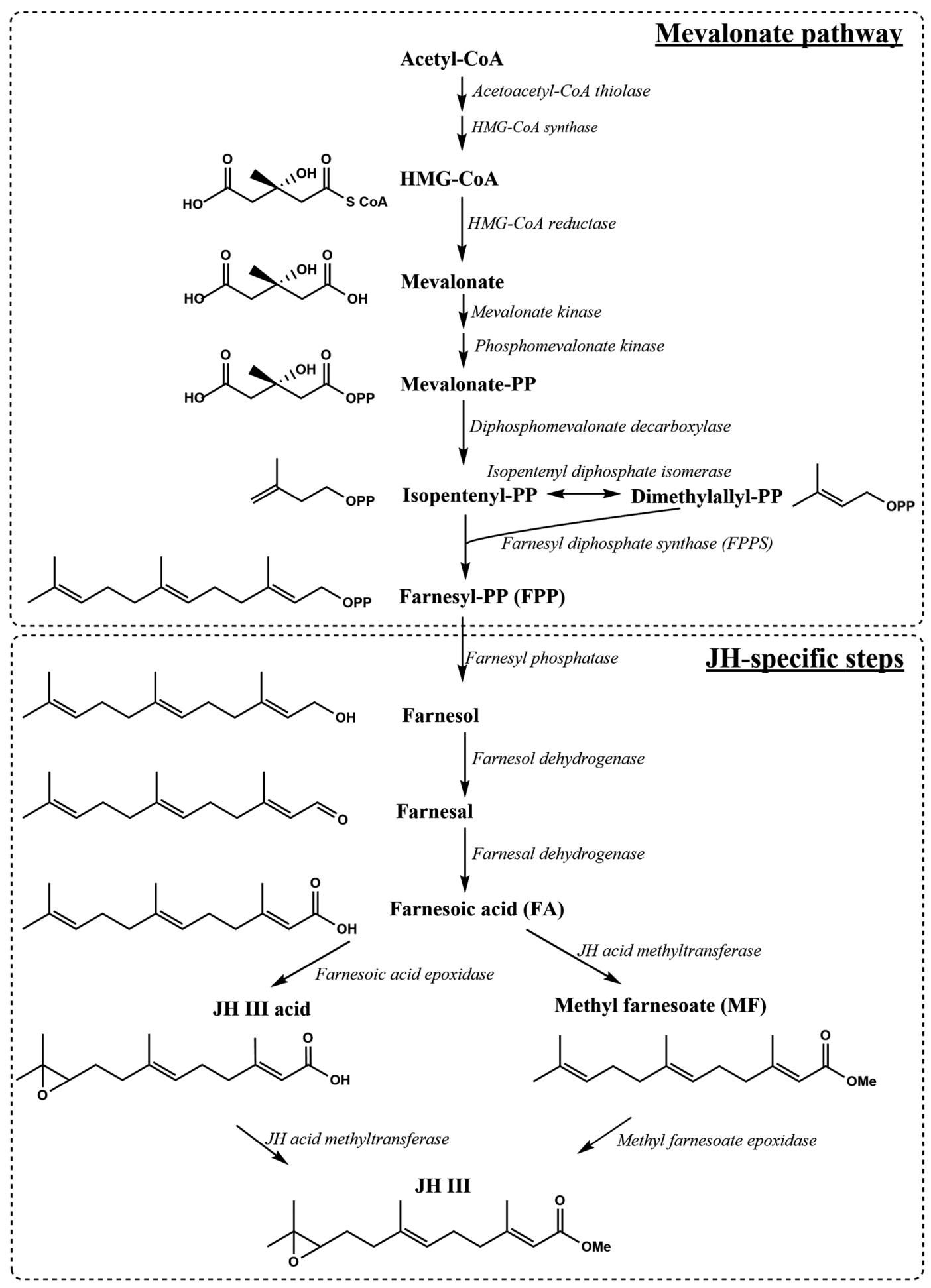

Fig. 1. Overview of the Juvenile Hormone Biosynthetic Pathway (Adapted from Goodman and Cusson ${ }^{5}$ ). This pathway is divided in two branches, the mevalonate pathway and the JH-specific steps. The order of the last two enzymatic steps may vary. Precursors are in bold and enzymes are in italics.

which allows induction of metamorphosis by ecdysteroids. ${ }^{8)}$ Termination of feeding followed by precocious metamorphosis is the typical response observed following an experimentally induced decline in the JH titer prior to the last larval stadium, resulting in the formation of malformed pupae or miniature adults that may not be able to reproduce or survive. Inhibition of JH synthesis has therefore been considered a promising avenue in the development of so-called third-generation insecticides. ${ }^{4}$

FPPS had initially not been viewed as an ideal target for the design of insect pest control products, largely because of its ubiq- 
uity in Nature. However, lepidopteran FPPS was soon observed to display properties suggesting it had unique structural features potentially amenable to lepidopteran-specific inhibition. ${ }^{9-11)}$ Here, we review recent advances in the characterization of FPPS and offer insights into the rational design of effective and selective inhibitors.

\section{Ubiquitous Nature of FPPS}

Considering its pivotal position in the MVP (Fig. 1), with its product serving as precursor to numerous molecules, FPPS has become the most extensively studied short-chain isoprenyl diphosphate synthase (prenyltransferase). FPPS catalyzes the condensation of dimethylallyl diphosphate (DMAPP; $\mathrm{C}_{5}$ ) and isopentenyl diphosphate (IPP; $\mathrm{C}_{5}$ ) in order to form the intermediate geranyl diphosphate (GPP; $\mathrm{C}_{10}$ ), which then undergoes a second condensation step with IPP that results in the formation of farnesyl diphosphate (FPP; $\mathrm{C}_{15}$ ). In addition to being used directly for protein farnesylation, the FPP molecule is used as backbone for the synthesis of other sesquiterpenoids and as building block in the biosynthesis of larger terpenoids such as diterpenes, dolichols, mitochondrial ubiquinones and sterols. In most organisms, the principal FPP-derived end-products are sterols, including ergosterol and cholesterol. However, since insects do not produce sterols, FPP is used in the production of alternative end-products such as $\mathrm{JH}$.

FPPSs have been purified from a variety of organisms, including the yeast Saccharomyces cerevisiae, ${ }^{12)}$ chicken, ${ }^{13)}$ pig, ${ }^{14)}$ human, ${ }^{15)}$ and green pepper Capsicum annuum, ${ }^{16)}$ to name a few. These purified FPPSs have all been found to form homodimers, with each subunit ranging in size from 32 to $44 \mathrm{kDa}$. Similarly, FPPS cDNAs have been cloned from various organisms. ${ }^{17-25)}$ In all cases the proteins are predicted to be active as homodimers, in contrast to several prenyltransferases that require other protein subunits for activity.

Significant structural information is available through X-ray crystallography, site-directed mutagenesis, and microcalorimetry studies on FPPS enzymes. The FPPS protein has seven highly conserved peptide domains (I-VII) made up of 10 helices, with two specific regions containing aspartate-rich motifs (DDxxD, where $\mathrm{x}$ represents any amino acid) important for sub- strate binding and catalysis. ${ }^{26,27)}$ The first aspartate-rich motif (FARM) is present in domain II (helix D) while the second one (SARM) is located in domain VI (helix H), followed by a conserved lysine residue downstream. In its unreactive form, the FARM and SARM motifs are facing each other on opposite walls of the central cavity, $\sim 12 \AA$ apart. Upon allylic substrate binding structural changes occur to cause the FARM and SARM to move towards one another and to complete substrate binding interactions needed for catalysis. The first crystal structure of avian FPPS $^{28)}$ confirmed that the enzyme is comprised of two subunits forming a tightly coupled homodimeric structure, where each monomer has an allylic binding site for DMAPP or GPP.

The chain-length specificity of FPPSs is largely dependent on the FARM and the region immediately preceding it. Based on this, FPPSs can be divided in two categories that also differentiate eukaryotic (category I) and eubacterial FPPSs (category II), respectively. ${ }^{29,30)}$ Eukaryotic FPPSs differ from the eubacterial enzymes by having two aromatic residues (typically Phe or Tyr) -4 and -5 from the FARM, known as the chain-length determination region (CLD), which provide a floor to the allylic binding site restricting binding of larger chain length species. While the basis of their catalytic mechanism requires the presence of divalent cations (generally $\mathrm{Mg}^{2+}$ or $\mathrm{Mn}^{2+}$ ), work on some short-chain isoprenyl diphosphate synthases, namely in the juvenile horseradish leaf beetle Phaedon cochleariae, ${ }^{31)}$ the mosquito Aedes aegyp$t i{ }^{32)}$ and the hardy rubber tree Eucomma elmoides, ${ }^{33)}$ suggests that carbon chain-length determination $\left(\mathrm{C}_{10} v s . \mathrm{C}_{15}\right.$ products $)$ could also be regulated by the concentrations and species of the metal cofactor $\left(\mathrm{Mn}^{2+}, \mathrm{Mg}^{2+}\right.$ and $\left.\mathrm{Co}^{2+}\right)$ present.

\section{Catalytic Mechanism of Eukaryotic FPPSs}

Eukaryotic FPPSs catalyze a reaction that results in the connection of two units of IPP to DMAPP following a mechanism referred to as "head-to-tail" condensation, which is a sequential process of ionization-condensation-elimination (Fig. 2). A three-step electrophilic alkylation is responsible for the elongation. The first step involves the breakage of the $\mathrm{C}-\mathrm{O}$ bond of DMAPP to form a tight ion pair intermediate $\left(\mathrm{DMA}^{+} / \mathrm{PPi}^{-}\right)$. The dimethylallyl carbocation alkylates the si face of the C3-C4 double bond of IPP, generating a second carbocation intermediate.

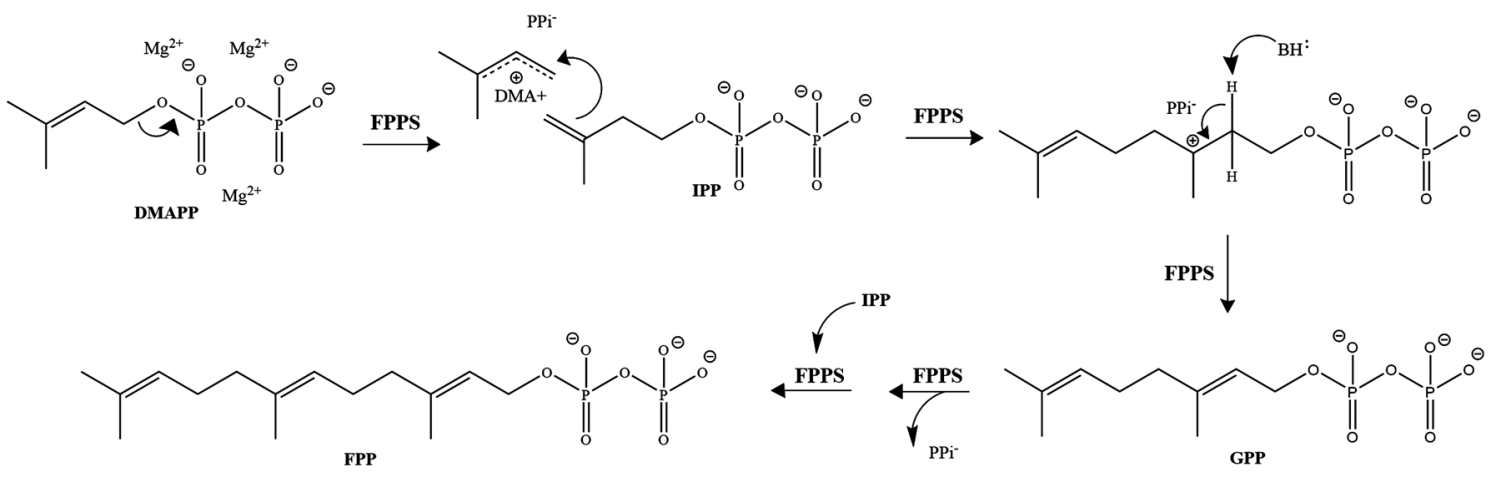

Fig. 2. Mechanism of "head-to-tail" condensation in eukaryotic FPPSs. For detail, see the main text. 
Stereospecific deprotonation of C2 pro- $R$ hydrogen of IPP by a catalytic base $(\mathrm{BH})$ causes the formation of a new trans $\mathrm{C} 2-\mathrm{C} 3$ double bond, resulting in the intermediate product GPP. A rearrangement inside the catalytic cavity induced by the enzymeGPP complex repositions GPP in the allylic substrate binding site and releases the diphosphate group before the second headto-tail condensation takes place, generating the final product E,E-FPP. ${ }^{34,35)}$

\section{Role of FPPS in Insect JH Biosynthesis}

Eight different structural forms of $\mathrm{JH}$ have been identified in insects so far, all of which are derived from the sesquiterpene $\left(\mathrm{C}_{15}\right)$ precursor, farnesyl diphosphate (FPP) or one of its homologs. Up to the point of FPP formation, JH biosynthetic enzymes are homologous to those found in all living organisms that use the mevalonate pathway; the subsequent steps are JH-specific. Four structural forms of $\mathrm{JH}$ (JH 0, 4-methyl-JH I, JH I and JH II) are specific to the Lepidoptera (Fig. 3). They bear one to three ethyl branches originating from the substitution of propionyl-CoA for one of three acetyl-CoAs at an early step of the MVP/JH biosynthetic pathway. ${ }^{36)}$ The substrate specificity of FPPS in relation to $\mathrm{JH}$ homologs has been examined. Compared to FPPS of pig liver, one of two FPPSs purified from Bombyx mori displayed a higher coupling efficiency for the precursors of $\mathrm{JH} 0$ and for coupling with homologous IPP (HIPP). ${ }^{9)}$ Prenyltransferase derived from homogenates of lepidopteran CA has been shown to be involved in the biosynthesis of the ethyl-substituted JHs and to display substrate/product selectivity favoring bulkier ethyl-branched ligands. ${ }^{11)}$ It has been suggested that these taxon-specific features could be exploited for the development of
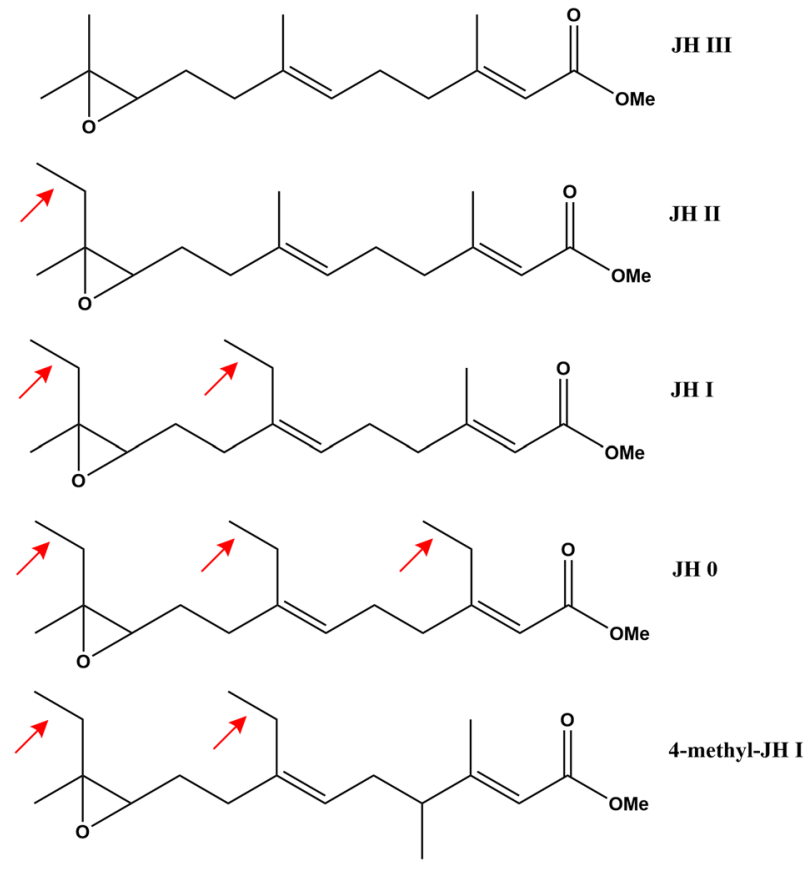

Fig. 3. Structures of the known lepidopteran juvenile hormones, four of which feature ethyl branches (arrows).
Lepidoptera-specific inhibitors with insecticidal properties. ${ }^{21,37)}$

\section{Lepidopteran Genomes Feature at Least Two Distinct FPPS Paralogs}

FPPS is encoded by a variable number of copies of associated genes. While FPPS is a single-copy gene in humans, plants typically have two copies of FPPS, with alternate splicing producing up to three different isoforms whose expression varies as a function of location, development, and environmental conditions. ${ }^{38)}$ In B. mori, transcripts of all MVP enzymes but one are most abundant in the $\mathrm{CA}$, the major organ of $\mathrm{JH}$ synthesis and release, as compared to other tissues. ${ }^{39,40)}$ Similarly, transcript analysis of MVP enzymes and those that are specific to late steps in $\mathrm{JH}$ biosynthesis (i.e., farnesol dehydrogenase, $\mathrm{JH}$ acid methyl transferase, and JH epoxidase) indicates that FPPS is highly specific to larval CA. ${ }^{41)}$ FPPS is one of the most extensively studied enzymes of the insect MVP and has been identified/characterized in many insects. The first cloning of an insect FPPS cDNA was that of Castillo-Garcia and Couillaud in 1999. ${ }^{19)}$ Since then, many additional insect FPPSs have been isolated. ${ }^{42)}$ Typically, insect FPPS is present as a single-copy gene, but there are notable exceptions including the honey bee, Apis mellifera, whose genome contains six putative FPPS paralogs ${ }^{43,44)}$ presumed to be associated with a variety of biochemical pathways. Likewise, with one exception, ${ }^{45)}$ the genomes of aphid species examined to date encode two FPPS paralogs, similar to one another in their primary sequences. ${ }^{42)}$ The two aphid FPPS paralogs display an important substitution in their CLD region compared with the majority of other known eukaryotic FPPSs. While the FARM sequence itself (DDxxD) is conserved in aphid FPPSs, a Gln residue substitutes for Phe/Tyr at position $-4 .{ }^{46-48)}$ Vandermoten et al. ${ }^{49)}$ performed site-directed mutagenesis and in vitro assays using recombinant FPPS, showing that this substitution plays a role in the bifunctional behavior of the aphid enzyme, which was uncovered by the same group in 2008. ${ }^{47)}$ As such, this enzyme could produce significant amounts of both GPP and FPP in the presence of DMAPP and $\left[{ }^{14} \mathrm{C}\right] \mathrm{IPP}$. This hypothesis is also supported by the work of Lewis et al., ${ }^{46)}$ where monoterpenes and sesquiterpenes were produced using a linked assay in which the aphid FPPS was in the presence of either a monoterpene or sesquiterpene synthase. It seems aphids have evolved an enzyme able to generate both GPP and FPP, since no additional putative geranyl diphosphate synthase (GPPS) could be found in the pea aphid genome. ${ }^{47)}$ GPP is used as a precursor for sex pheromone and FPP is the precursor of both an alarm pheromone and $\mathrm{JH}$. While only one of the two recombinant aphid FPPSs was active in vitro, they display an identical CLD region sequence, which suggests a shared bifunctional behavior. ${ }^{47)}$

In the Lepidoptera, two very distinct paralogs, referred to as type- 1 and type-2 FPPS, were found in several species. ${ }^{21,22,39,50)}$ Interestingly, the $B$. mori genome was observed to contain two copies of the type-2 paralog (BmFPPS2 and BmFPPS3), both expressed predominantly in the CA. ${ }^{39)}$ This observation may not be an isolated case inasmuch as the genomes of Manduca sexta 


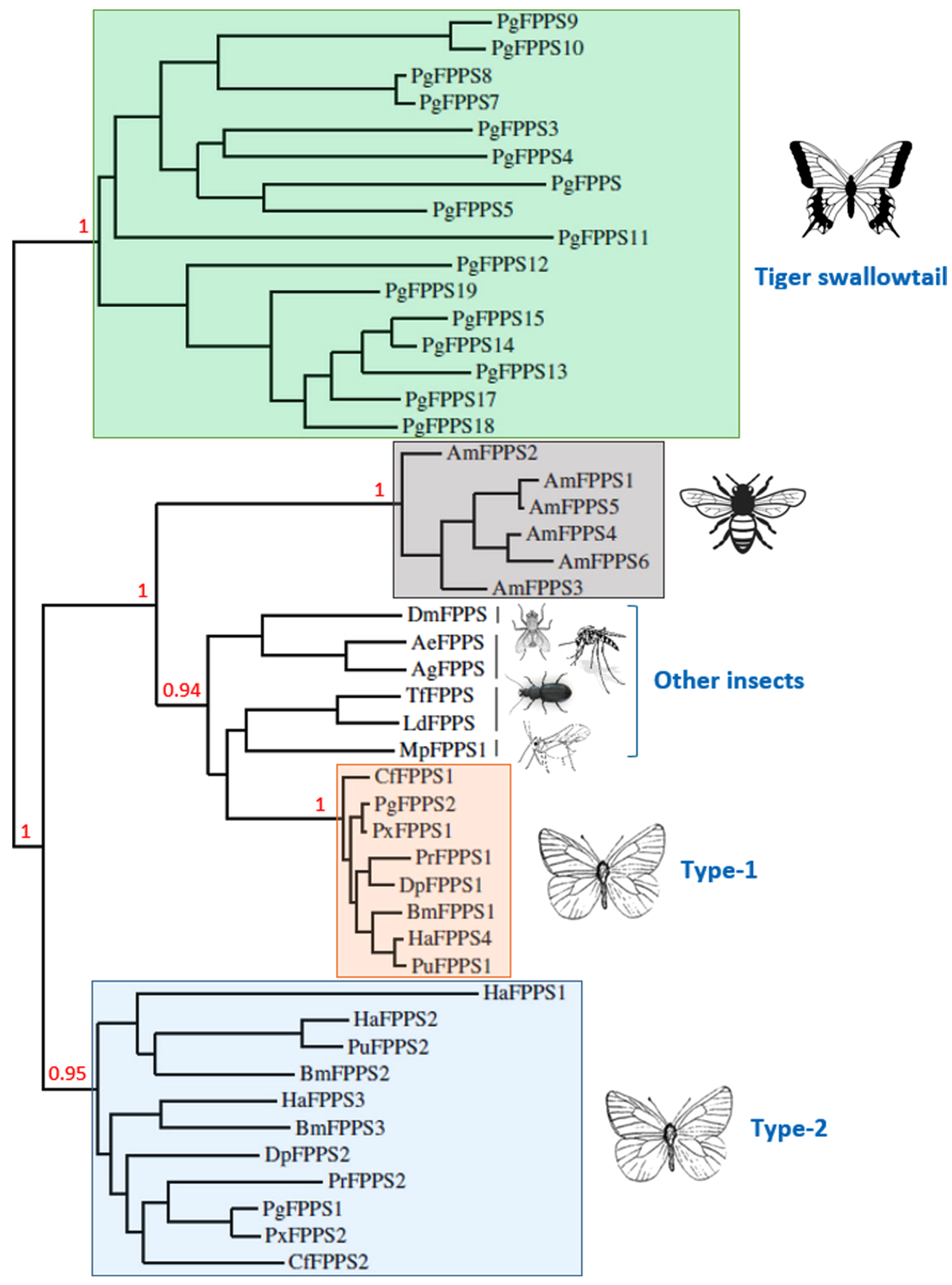

Fig. 4. Maximum likelihood tree for a selection of insect FPPSs. The tree was constructed using the PhyML tool (http://www.phylogeny.fr), after alignment of the amino acid sequences using MUSCLE (https://www.ebi.ac.uk/Tools/msa/muscle/). Branch support was assessed using the approximate Likelihood-Ratio Test functionality of PhyML. The tree was drawn using TreeDyn (http://www.treedyn.org/). Species, FPPS labels and accession numbers (except for Papilio glaucus): Aedes aegypti, AeFPPS: XP_001663796.1. Anopheles gambiae, AgFPPS: XP_308653.4. Apis mellifera, AmFPPS1: XP_001122575.3; AmFPPS2: XP_026300402.1; AmFPPS3: XP_006572097.2; AmFPPS4: XP_026300405.1; AmFPPS5: XP_624298.3; AmFPPS6: XP_016772838.2. Bombyx mori, BmFPPS1: BAB69490.1; BmFPPS2: NP_001093301.1; BmFPPS3: NP_001093302.1. Choristoneura fumiferana, CfFPPS1: AAY26575.1; CfFPPS2: AAY33485.1. Danaus plexipus, DpFPPS1: OWR45492.1; DpFPPS2: OWR45491.1. Drosophila melanogaster, DmFPPS: NP_477380.1. Helicoverpa armigera, HaFPPS1: XP_021182121.1; HaFPPS2: XP_021182149.1; HaFPPS3: XP_021182134.1; HaFPPS4: XP_021182148.1. Leptinotarsa decemlineata, LdFPPS: XP_023019940.1. Myzus persicae, MpFPPS1: AAY33491.1. Papilio glaucus (IDs from official gene set; see Table S4F in ${ }^{47}$ ), PgFPPS1: pg1854.12; PgFPPS2: pg1854.13; PgFPPS3: pgl854.9; PgFPPS4: pg1854.11; PgFPPS5: pgl10805.1; PgFPPS6: pg1854.10; PgFPPS7: pgl123.5; PgFPPS8: pgl123.3; PgFPPS9: pg13843.1; PgFPPS10: pgl3843.2; PgFPPS11: pgl1544.3; PgFPPS12: pgl10805.9; PgFPPS13: pg12397.1; PgFPPS14: pgl2898.1; PgFPPS15: pgl2898.3; PgFPPS17: pgl2898.5; PgFPPS18: pgl10805.8; PgFPPS19: pgl2898.4. Papilio xuthus: PxFPPS1: KPJ05697.1; PxFPPS2: KPJ05696.1. Pieris rapae: PrFPPS1: XP_022112553.1; PrFPPS2: XP_022112560.1. Pseudaletia (Mythimna) unipuncta: PuFPPS1: AAY33487.1; PuFPPS2: unpublished. Tetropium fuscum: TfFPPS: AFR31785.1. 
and Helicoverpa armigera feature two and three isoforms of the type-2 FPPS, respectively (Fig. 4), a phenomenon that is likely the result of a gene duplication event. Recently, Cong et al. ${ }^{51)}$ reported a major expansion of the FPPS gene family in the genus Papilio where 16 putative FPPS paralogs, in addition to the type1 and type-2 FPPSs, were discovered in the genomes of these butterflies. Because these new paralogs form a very distinct cluster in an FPPS gene tree (Fig. 4), resulting from a shortened Helix D and modified FARM, it is unclear whether they function as FPPSs or have another prenyltransferase function.

When compared to "conventional" eukaryotic FPPSs, lepidopteran type-1 FPPSs display several active site substitutions, most notably in the FARM region, where NDxxE substitutes for DDxxD. These substitutions are expected to have an impact on catalysis, based on site-directed mutagenesis done on other FPPSs, which shows that the first and last aspartates are essential for catalytic activity, ${ }^{26,27)}$ and crystallographic studies confirm that these residues are involved in metal coordination. ${ }^{52)}$ In addition, His and Gln substitute for the typical aromatic residues at positions -5 and -4 relative to the FARM, creating a larger cavity floor with additional hydrogen bonding capabilities. The fact that aphid and lepidopteran FPPSs both show taxon-specific active-site features makes them promising in terms of targetspecific inhibitor design. Since FPPS paralogs are likely involved in other isoprenoid pathways beside JH biosynthesis, their inhibition could result in multiple physiological disturbances.

\section{FPPS2 is the Likely Paralog Involved in Lepidopteran JH Biosynthesis}

It has long been hypothesized that one or more enzymes responsible for lepidopteran JH biosynthesis exhibit structural features allowing them to accommodate bulkier homologous substrates and products used as precursors of ethyl-branched JHs, ${ }^{36,53)}$ with FPPS playing a key role. ${ }^{54)}$ In fact, homologous substrates appeared selectively favored by the endogenous enzyme in prenyltransferase assays conducted on $M$. sexta CA homogenates. ${ }^{10)}$ This has been corroborated by studies where the lepidopteran enzyme was shown to display greater steric latitude around the C-3 and C-7 alkyl position of DMAPP and GPP, compared with pig liver FPPS. ${ }^{55)}$ When comparing the sequences and homology models of type- 1 and type-2 FPPSs (FPPS1 and FPPS2), the type-2 enzyme has a more "conventional" active site, though variable non-aromatic residues at position -4 from the FARM have been observed. Initial docking studies showed FPPS1 as having greater binding affinity for homologous GPP substrates and FPP products. However, transcriptional analysis of type- 1 and type-2 FPPSs in an array of B. mori tissues showed a ubiquitous distribution for BmFPPS1 and a confinement to the CA for BmFPPS2, ${ }^{21,39)}$ which suggested that the principal prenyltransferase involved in $\mathrm{JH}$ biosynthesis in the Lepidoptera was type-2 FPPS. Similarly, $M$. sexta FPPS2 has been found to be specific to the CA, with transcript levels correlating with known prepupal JH biosynthesis. ${ }^{41)}$ Work where the two Choristoneura fumiferana FPPSs were produced as recombinant proteins and then tested for their ability to couple a variety of allylic substrates with radioactive co-substrate $\left[{ }^{14} \mathrm{C}\right] \mathrm{IPP}$ showed that CfFPPS1 was inactive in the presence of $\mathrm{Mn}^{2+}, \mathrm{Mg}^{2+}$, and several other metal cations and under a wide range of assay conditions, while CfFPPS2 had significant activity with the homologous allylic substrates. Interestingly, when both enzymes were put together, a synergistic effect was observed for the coupling of bishomogeranyl diphosphate and homogeranyl diphosphate with IPP. ${ }^{56)}$ While this outcome may indicate the involvement of a heterodimeric CfFPPS1-CfFPPS2 complex, the in vivo significance of this observation remains to be assessed. Transcriptional studies in B. mori indicated that both type- 1 and type-2 FPPS were present in the CA (a 1:20 BmFPPS1:BmFPPS2 ratio in larval $\mathrm{CA}^{21)}$ see also ${ }^{39)}$ ), supporting the possibility for heteromers to form in this tissue. In other tissues where BmFPPS1 transcripts were abundant, BmFPPS2 transcripts levels were close to the q-RT-PCR detection limit, pointing to a distinct role for FPPS1 in non-CA tissues. In addition to B. mori, two lepidopteran species have been observed to display elevated FPPS1 transcripts in Malpighian tubules; the role of this protein in this tissue has not yet been elucidated (Barbar, A. Cusson, M., Béliveau, C. Sen, S.E., unpublished results).

\section{Lepidopteran FPPS Inhibitor Development}

There are more than 200 crystal structures of FPPSs currently available in the Protein Data Bank (rcsb.org), a high proportion of which are for human FPPS bound to various ligands. Because of the importance of FPPS in sterol and ubiquinone biosynthesis and protein prenylation, human FPPS serves as a drug target for the treatment or prevention of certain diseases. In addition, FPPSs from several human parasites are being investigated as targets for inhibitors that could prevent the survival of organisms such as Trypanosoma cruzi, the causal agent of Chagas disease. $^{57)}$

The molecules most commonly considered for FPPS inhibition belong to a group known as bisphosphonates (BPs). Many BPs have been synthesized and evaluated in clinical trials for the treatment of specific human medical conditions; the best ones showing $\mathrm{IC}_{50}$ values in the low nanomolar range. Because of their high binding affinity for calcium, BPs have been highly useful in targeting bone pathologies such as osteoporosis, hypercalcemia, Paget's disease and certain cancers. These compounds mimic the diphosphate group found in natural allylic diphosphate substrates (Fig. 5). Instead of the central oxygen atom at the center of the phosphodiester, a carbon is substituted to provide chemical stability towards diphosphate hydrolysis. Usually, BPs designed for FPPS inhibition contain a nitrogen atom that can be protonated under physiological conditions or is alkylated to mimic the putative allylic carbocation intermediate formed during the rate-determining step. ${ }^{58)}$

A first series of compounds aimed at disrupting insect growth through FPPS inhibition was synthesized and evaluated by Sen et al. ${ }^{59)}$ It was hypothesized that appropriately $N$-alkylated BPs can provide selective binding to the C-3 binding region of 
<smiles>O=P(O)(O)OP(=O)(O)O</smiles>

Bisphosphonate<smiles>[R]C([R])(P(=O)(O)O)P(=O)(O)O</smiles>

Fig. 5. General chemical structure of the bisphosphonate group compared with pyrophosphate. The general chemical structure of $\mathrm{N}$-alkylated ortho-substituted pyridinium BPs is also shown on the right.

lepidopteran FPPS2, based on the observation that homologous allylic substrates such as HDMAPP and bishomogeranyl diphosphate (BHGPP, precursor to JH0 and JH1) contain C-3 alkyl substituents that are larger than in DMAPP and GPP, and because C-3 alkyl analogs of DMAPP were shown to be readily accommodated by the prenyltransferase of $M$. sexta CA. ${ }^{55)}$ Thus a series of $\mathrm{N}$-alkylated ortho-substituted pyridinium BPs (Fig. 5) were chosen as candidates for selective inhibition of FPPS2, following docking of potential structures in the active site of a homology model of spruce budworm (C. fumiferana) FPPS. Inhibitory effects of these molecules on the coupling of GPP to IPP by pig liver FPPS ( $\mathrm{IC}_{50}$ ranging from $0.2 \mu \mathrm{M}$ to no inhibition), and recombinant fly (Drosophila melanogaster; $\mathrm{IC}_{50}$ ranging from $49 \mu \mathrm{M}$ to no inhibition) and moth (CfFPPS2; $\mathrm{IC}_{50}$ ranging from 0.7 to $2.8 \mu \mathrm{M}$ ) FPPSs were shown to be more selective towards the lepidopteran protein. ${ }^{59)}$ These results are consistent with the notion that there is greater steric latitude in the active site of lepidopteran FPPS. Picard et al. ${ }^{60)}$ presented the first structural study of a lepidopteran FPPS (Fig. 6). C. fumiferana FPPS2 (CfFPPS2), either in the absence of ligand or in the presence of $\mathrm{N}$-methyl or $\mathrm{N}$-ethyl pyridinium BP inhibitors, with or without the co-substrate IPP, revealed differences between the FPPSs of moths and those of other organisms. Similar to other FPPSs, the BPs all bound to the allylic binding site of the protein. A comparison with human structures provided insight into the structural determinants that confer substrate specificity to CfFPPS2. Notably residues occupying positions -4 and -3 relative to the FARM, which form a hydrophobic pocket for allylic substrate binding, were observed to be smaller for the lepidopteran protein, and therefore important determinants for the binding of homologous substrates. In addition, unlike vertebrate and most other FPPSs, ${ }^{61)}$ IPP binding was not required for the final stiffening and closure of the C-terminal tail of CfFPPS2, a feature that could be relevant for selective inhibitor design since other FPPSs need IPP to close entry of the active site and limit solvent exposure. Interestingly, although the ortho-substituted pyridinium bisphosphonates bound to the allylic site as predicted, their alkyl groups were oriented towards the homoallylic binding site, with the bulkier propyl-substituted inhibitor penetrating deeply into the IPP binding pocket and thereby functioning as a bi-substrate inhibitor.

\section{Conclusion}

Lepidopteran FPPS is an attractive target for the development of bio-rational insecticides, particularly in view of its pivotal role as the final step of the mevalonate pathway. In addition to playing a role in juvenile hormone biosynthesis, its product, FPP, is used in metabolic pathways such as the biosynthesis of diterpenes, ubiquinones, dolichol, other sesquiterpenes, and the prenylation of proteins. When the catalytic activity of FPPS is disrupted, not only is JH biosynthesis interfered with, but other key processes may be inhibited as well, resulting in physiological disturbances potentially fatal to the target insect. The X-ray structures obtained for lepidopteran FPPS have provided vital information for the continued design of new inhibitors displaying enhanced target selectivity and greater inhibitory activity. We are currently designing and testing new FPPS inhibitors with a view to developing innovative pest-control products targeting



Fig. 6. Crystal structure of CfFPPS2. Top panel: homodimeric representation of CfFPPS2 with $N$-methyl pyridinium BP inhibitor (1b, purple sticks) and co-substrate IPP (magenta sticks) (PDB Code: 6B06). One subunit is shown in grey and the second subunit is shown in a rainbow color scheme indicating the $\mathrm{N}$-terminal (blue) and $\mathrm{C}$-terminal (red) regions. Bottom panel: closeup of $\mathbf{1 b}$ and IPP interactions with the receptor allylic and homoallylic binding sites. The aspartates from the FARM are shown in turquoise sticks while those from the SARM are shown in yellow sticks. 
the Lepidoptera.

\section{References}

1) C. M. Williams: Third-generation pesticides. Sci. Am. 217, 13-17 (1967).

2) M. Jindra, S. R. Palli and L. M. Riddiford: The juvenile hormone signaling pathway in insect development. Annu. Rev. Entomol. 58, 181204 (2013).

3) V. Smykal, T. Daimon, T. Kayukawa, K. Takaki, T. Shinoda and M. Jindra: Importance of juvenile hormone signaling arises with competence of insect larvae to metamorphose. Dev. Biol. 390, 221-230 (2014).

4) M. Cusson and S. R. Palli: Can juvenile hormone research help rejuvenate integrated pest management? Can. Entomol. 132, 263-280 (2000).

5) W. G. Goodman and M. Cusson: The juvenile hormones. In "Insect Endocrinology," ed. by L.I. Gilbert, Elsevier, London, pp. 310-365, 2012.

6) S. S. Tobe and B. Stay: Structure and regulation of the corpus allatum. Adv. Insect Physiol. 18, 305-432 (1985).

7) M. Cusson and J. N. McNeil: Involvement of juvenile hormone in the regulation of pheromone release activities in a moth. Science $\mathbf{2 4 3}$, 210-212 (1989).

8) L. M. Riddiford: Cellular and molecular actions of juvenile hormone. I. General considerations and premetamorphic actions. Adv. Insect Physiol. 24, 213-274 (1994).

9) T. Koyama, M. Matsubara and K. Ogura: Isoprenoid enzyme systems of silkworm. II: Formation of the juvenile hormone skeletons by farnesyl pyrophosphate synthase II. J. Biochem. 98, 457-463 (1985).

10) S. E. Sen, G. J. Ewing and N. Thursten: Characterization of lepidopteran prenyltransferase in Manduca sexta corpora allata. Arch. Insect Biochem. Physiol. 32, 315-332 (1996).

11) S. E. Sen and G. J. Ewing: Natural and unnatural terpenoid precursors of insect juvenile hormone. J. Org. Chem. 62, 3529-3536 (1997).

12) N. L. Eberhardt and H. C. Rilling: Prenyltransferase from Saccharomyces cerevisiae: Purification to homogeneity and molecular properties. J. Biol. Chem. 250, 863-866 (1975).

13) B. C. Reed and H. C. Rilling: Crystallization and partial characterization of prenyltransferase from avian liver. Biochemistry 14, 50-54 (1975).

14) L. S. Yeh and H. C. Rilling: Purification and properties of pig liver prenyltransferase: Interconvertible forms of the enzyme. Arch. Biochem. Biophys. 183, 718-725 (1977).

15) G. F. Barnard and G. Popjak: Human liver prenyltransferase and its characterization. Biochim. Biophys. Acta 661, 87-99 (1981).

16) P. Hugueney and B. Camara: Purification and characterization of farnesyl pyrophosphate synthase from Capsicum annuum. FEBS Lett. 273, 235-238 (1990).

17) C. F. Clarke, R. D. Tanaka, K. Svenson, M. Wamsley, A. M. Fogelman and P. A. Edwards: Molecular cloning and sequence of a cholesterolrepressible enzyme related to prenyltransferase in the isoprene biosynthetic pathway. Mol. Cell. Biol. 7, 3138-3146 (1987).

18) D. J. Wilkin, S. Y. Kutsunai and P. A. Edwards: Isolation and sequence of the human farnesyl pyrophosphate synthetase cDNA. J. Biol. Chem. 265, 4607-4614 (1990).

19) M. Castillo-Gracia and F. Couillaud: Molecular cloning and tissue expression of an insect farnesyl diphosphate synthase. Eur. J. Biochem. 262, 365-370 (1999).

20) K. Kikuchi, M. Hirai and T. Shiotsuki: Molecular cloning and tissue distribution of farnesyl pyrophosphate synthase from the silkworm. Sanshi Konchuu Baiotekku 70, 167-172 (2001).

21) M. Cusson, C. Béliveau, S. E. Sen, S. Vandermoten, R. J. Rutledge, D. Stewart, F. Francis, É. Haubruge, P. Rehse, D. J. Huggins, A. P. G. Dowling and G. H. Grant: Characterization and tissue-specific expression of two lepidopteran farnesyl diphosphate synthase homologs: Implications for the biosynthesis of ethyl-substituted juvenile hormones. Proteins 65, 742-758 (2006).

22) S. E. Sen, C. Trobaugh, C. Béliveau, T. Richard and M. Cusson: Cloning, expression and characterization of a dipteran farnesyl diphosphate synthase. Insect Biochem. Mol. Biol. 37, 1198-1206 (2007).

23) M. S. Anderson, J. G. Yarger, C. L. Burck and C. D. Poulter: Farnesyl diphosphate synthase: Molecular cloning, sequence, and expression of an essential gene from Saccharomyces cerevisiae. J. Biol. Chem. 264, 19176-19184 (1989).

24) N. Cunillera, M. Arró, D. Delourme, F. Karst, A. Boronat and A. Ferrer: Arabidopsis thaliana contains two differentially expressed farnesyl-diphosphate synthase genes. J. Biol. Chem. 271, 7774-7780 (1996).

25) A. Montalvetti, A. Fernandez, J. M. Sanders, S. Ghosh, E. Van Brussel, E. Oldfield and R. Docampo: Farnesyl pyrophosphate synthase is an essential enzyme in Trypanosoma brucei: In vitro RNA interference and in vivo inhibition studies. J. Biol. Chem. 278, 1707517083 (2003).

26) A. Joly and P. A. Edwards: Effect of site-directed mutagenesis of conserved aspartate and arginine residues upon farnesyl diphosphate synthase activity. J. Biol. Chem. 268, 26983-26989 (1993).

27) L. Song and C. D. Poulter: Yeast farnesyl-diphosphate synthase: Sitedirected mutagenesis of residues in highly conserved prenyltransferase domains I and II. Proc. Natl. Acad. Sci. U.S.A. 91, 3044-3048 (1994).

28) L. C. Tarshis, P. J. Proteau, B. A. Kellogg, J. C. Sacchettini and C. D. Poulter: Regulation of product chain length by isoprenyl diphosphate synthases. Proc. Natl. Acad. Sci. U.S.A. 93, 15018-15023 (1996).

29) H. Hemmi, M. Noike, T. Nakayama and T. Nishino: An alternative mechanism of product chain-length determination in type III geranylgeranyl diphosphate synthase. Eur. J. Biochem. 270, 2186-2194 (2003).

30) K. Wang and S. Ohnuma: Chain-length determination mechanism of isoprenyl diphosphate synthases and implications for molecular evolution. Trends Biochem. Sci. 24, 445-451 (1999).

31) S. Frick, R. Nagel, A. Schmidt, R. R. Bodemann, P. Rahfeld, G. Pauls, W. Brandt, J. Gershenzon, W. Boland and A. Burse: Metal ions control product specificity of isoprenyl diphosphate synthases in the insect terpenoid pathway. Proc. Natl. Acad. Sci. U.S.A. 110, 4194-4199 (2013).

32) C. Rivera-Perez, P. Nyati and F. G. Noriega: A corpora allata farnesyl diphosphate synthase in mosquitoes displaying a metal ion dependent substrate specificity. Insect Biochem. Mol. Biol. 64, 44-50 (2015).

33) H. Kajiura, N. Suzuki, Y. Tokumoto, T. Yoshizawa, S. Takeno, K. Fujiyama, Y. Kaneko, H. Matsumura and Y. Nakazawa: Two Eucommia farnesyl diphosphate synthases exhibit distinct enzymatic properties leading to end product preferences. Biochimie 139, 95-106 (2017).

34) J. W. Cornforth, R. H. Cornforth, G. Popjak and L. Yengoyan: Studies on the biosynthesis of cholesterol. XX. Steric course of decarboxylation of 5-pyrophosphomevalonate and of the carbon to carbon bond formation in the biosynthesis of farnesyl pyrophosphate. J. Biol. Chem. 241, 3970-3987 (1966).

35) C. D. Poulter, J. C. Argyle and E. A. Mash: Farnesyl pyrophosphate synthetase. Mechanistic studies of the $1^{\prime}-4$ coupling reaction with 
2-fluorogeranyl pyrophosphate. J. Biol. Chem. 253, 7227-7233 (1978).

36) D. A. Schooley, K. J. Judy, B. J. Bergot, M. S. Hall and J. B. Siddall: Biosynthesis of the juvenile hormones of Manduca sexta: labeling pattern from mevalonate, propionate, and acetate. Proc. Natl. Acad. Sci. U.S.A. 70, 2921-2925 (1973).

37) M. Cusson, S. E. Sen and T. Shinoda: Juvenile hormone biosynthetic enzymes as targets for insecticide discovery. In "Advanced Technologies for Managing Insect Pests," eds. by I. Ishaaya, S.R. Palli and A.R. Horowitz, Springer, New York, pp. 31-55, 2012.

38) D. Guo, H. L. Li and S. Q. Peng: Structure conservation and differential expression of farnesyl diphosphate synthase genes in Euphorbiaceous plants. Int. J. Mol. Sci. 16, 22402-22414 (2015).

39) T. Kinjoh, Y. Kaneko, K. Itoyama, K. Mita, K. Hiruma and T. Shinoda: Control of juvenile hormone biosynthesis in Bombyx mori: Cloning of the enzymes in the mevalonate pathway and assessment of their developmental expression in the corpora allata. Insect Biochem. Mol. Biol. 37, 808-818 (2007).

40) H. Ueda, T. Shinoda and K. Hiruma: Spatial expression of the mevalonate enzymes involved in juvenile hormone biosynthesis in the corpora allata in Bombyx mori. J. Insect Physiol. 55, 798-804 (2009).

41) Y. Yin, Y.-W. Qiu, J. Huang, S. S. Tobe, S.-S. Chena and Z.-P. Kai: Enzymes in the juvenile hormone biosynthetic pathway can be potential targets for pest control. Pest Manag. Sci. 76, 1071-1077 (2020).

42) S. Vandermoten, É. Haubruge and M. Cusson: New insights into short-chain prenyltransferases: Structural features, evolutionary history and potential for selective inhibition. Cell. Mol. Life Sci. 66, 3685-3695 (2009).

43) Honeybee Genome Sequencing Consortium: Insights into social insects from the genome of the honeybee Apis mellifera. Nature 443, 931-949 (2006).

44) D. Cheng, M. Meng, J. Peng, W. Qian, L. Kang and Q. Xia: Genomewide comparison of genes involved in the biosynthesis, metabolism, and signaling of juvenile hormone between silkworm and other insects. Genet. Mol. Biol. 37, 444-459 (2014).

45) G. Y. Ma, X. F. Sun, Y. L. Zhang, Z. X. Li and Z. R. Shen: Molecular cloning and characterization of a prenyltransferase from the cotton aphid, Aphis gossypii. Insect Biochem. Mol. Biol. 40, 552-561 (2010).

46) M. J. Lewis, I. M. Prosser, A. Mohib and L. M. Field: Cloning and characterisation of a prenyltransferase from the aphid Myzus persicae with potential involvement in alarm pheromone biosynthesis. Insect Mol. Biol. 17, 437-443 (2008).

47) S. Vandermoten, B. Charloteaux, S. Santini, S. E. Sen, C. Béliveau, M. Vandenbol, F. Francis, R. Brasseur, M. Cusson and É. Haubruge: Characterization of a novel aphid prenyltransferase displaying dual geranyl/farnesyl diphosphate synthase activity. FEBS Lett. 582, 19281934 (2008).

48) Y. L. Zhang and Z. X. Li: Two different farnesyl diphosphate synthase genes exist in the genome of the green peach aphid, Myzus persicae. Genome 51, 501-510 (2008).

49) S. Vandermoten, S. Santini, E. Haubruge, F. Heuze, F. Francis, R.
Brasseur, M. Cusson and B. Charloteaux: Structural features conferring dual geranyl/farnesyl diphosphate synthase activity to an aphid prenyltransferase. Insect Biochem. Mol. Biol. 39, 707-716 (2009).

50) S. Zhan, C. Merlin, J. L. Boore and S. M. Reppert: The Monarch butterfly genome yields insights into long-distance migration. Cell 147, 1171-1185 (2011).

51) Q. Cong, D. Borek, Z. Otwinowski and N. V. Grishin: Tiger swallowtail genome reveals mechanisms for speciation and caterpillar chemical defense. Cell Rep. 10, 910-919 (2015).

52) K. L. Kavanagh, K. Guo, J. E. Dunford, X. Wu, S. Knapp, F. H. Ebetino, M. J. Rogers, R. G. Russell and U. Oppermann: The molecular mechanism of nitrogen-containing bisphosphonates as antiosteoporosis drugs. Proc. Natl. Acad. Sci. U.S.A. 103, 7829-7834 (2006).

53) M. G. Peter, P. D. Shirk, K. H. Dahm and H. Roller: On the specificity of juvenile hormone biosynthesis in the male Cecropia moth. Naturforsch 36, 579-585 (1981).

54) M. Cusson, A. Le Page, J. N. McNeil and S. S. Tobe: Rate of isoleucine metabolism in lepidopteran corpora allata: regulation of the proportion of juvenile hormone homologs released. Insect Biochem. Mol. Biol. 26, 195-201 (1996).

55) S. E. Sen, J. R. Hitchcock, J. L. Jordan and T. Richard: Juvenile hormone biosynthesis in $M$. sexta: Substrate specificity of insect prenyltransferase utilizing homologous diphosphate analogs. Insect Biochem. Mol. Biol. 36, 827-834 (2006).

56) S. E. Sen, M. Cusson, C. Trobaugh, C. Béliveau, T. Richard, W. Graham, A. Mimms and G. Roberts: Purification, properties and heteromeric association of type-1 and type-2 lepidopteran farnesyl diphosphate synthases. Insect Biochem. Mol. Biol. 37, 819-828 (2007).

57) S. B. Gabelli, J. S. McLellan, A. Montalvetti, E. Oldfield, R. Docampo and L. M. Amzel: Structure and mechanism of the farnesyl diphosphate synthase from Trypanosoma cruzi: Implications for drug design. Proteins 62, 80-88 (2006).

58) M. B. Martin, W. Arnold, H. T. Heath 3rd, J. A. Urbina and E. Oldfield: Nitrogen-containing bisphosphonates as carbocation transition state analogs for isoprenoid biosynthesis. Biochem. Biophys. Res. Commun. 263, 754-758 (1999).

59) S. E. Sen, L. Wood, R. Jacob, A. Xhambazi, B. Pease, A. Jones, T. Horsfield, A. Lin and M. Cusson: Disruption of insect isoprenoid biosynthesis with pyridinium bisphosphonates. Insect Biochem. Mol. Biol. 63, 113-123 (2015).

60) M.-È. Picard, A. Nisole, C. Béliveau, S. E. Sen, A. Barbar, R. Shi and M. Cusson: Structural characterization of a lepidopteran type-II farnesyl diphosphate synthase from the spruce budworm, Choristoneura fumiferana: implications for inhibitor design. Insect Biochem. Mol. Biol. 92, 84-92 (2018).

61) J. Park, Y. S. Lin, J. W. De Schutter, Y. S. Tsantrizos and A. M. Berghuis: Ternary complex structures of human farnesyl pyrophosphate synthase bound with a novel inhibitor and secondary ligands provide insights into the molecular details of the enzyme's active site closure. BMC Struct. Biol. 12, 32 (2012). 